\title{
Sex Differences in Associations of Cognitive Function with Perceptions of Pain in Older Adults
}

\author{
Raymond R. Romano ${ }^{\mathrm{a}, *}$, Alison R. Anderson ${ }^{\mathrm{a}}$, Michelle D. Failla ${ }^{\mathrm{f}}$, Mary S. Dietrich ${ }^{\mathrm{a}, \mathrm{b}, \mathrm{c}}$, \\ Sebastian Atalla ${ }^{\mathrm{a}}$, Michael A. Carter ${ }^{\mathrm{d}}$ and Todd B. Monroe ${ }^{\mathrm{e}}$ \\ ${ }^{a}$ School of Nursing, Vanderbilt University, Nashville, TN, USA \\ ${ }^{\mathrm{b}}$ Department of Biostatistics, Vanderbilt University Medical Center, Nashville, TN, USA \\ ${ }^{\mathrm{c}}$ Department of Psychiatry and Behavioral Science, Vanderbilt University Medical Center, Nashville, TN, USA \\ ${ }^{\mathrm{d}}$ College of Nursing, University of Tennessee Health Science Center, Memphis, TN, USA \\ ${ }^{\mathrm{e}}$ College of Nursing, The Ohio State University, Columbus, OH, USA \\ ${ }^{\mathrm{f}}$ Department of Psychiatry at Vanderbilt University Medical Center, Nashville, TN, USA
}

Accepted 14 May 2019

\begin{abstract}
.
Background: Sex differences in pain have been shown to exist in older adults with normal cognition and people with Alzheimer's disease. It is unknown if sex differences in pain in older adults exist in a range of communicative older adults with varying cognitive ability from no impairment to moderately severe cognitive impairment.

Objective: This study proposes to compare the association between psychophysical responses to experimental thermal pain between males and females to determine if sex differences in pain exist across the cognitive spectrum.

Methods: We conducted a secondary analysis of data from an age- and sex-matched between-groups cross-sectional study examining the psychophysical response to contact heat in people with and without dementia.

Results: Median age of males $(n=38)$ and females $(n=38)$ was 73 (range: $68-87)$ with similar distributions of MiniMental State Examination (MMSE) scores (range: 11-30). Findings revealed inverse statistically significant associations with the threshold temperature of warmth (females: $r=-0.41, p=0.010$; males: $r=-0.33, p=0.044$ ). There was an apparent divergent pattern of MMSE associations with unpleasantness ratings between the groups. At the moderate pain threshold, that difference became statistically significant $(p=0.033)$. Females demonstrated a positive association of MMSE with unpleasantness $(r=0.30, p=0.072)$, while males demonstrated an inverse association at that respective threshold $(r=-0.20$, $p=0.221)$.

Conclusions: Between-group findings suggest that patterns of responses to thermal stimulus intensity may differ between males and females with worsening cognition with females reporting significantly less unpleasantness with the percept of moderate pain and males reporting significantly higher unpleasantness with moderate pain perception.
\end{abstract}

Keywords: Cognition, dementia, pain, sex differences

\section{INTRODUCTION}

Nearly 50 million adults worldwide over the age of 65 years will develop dementia [1,2], and

\footnotetext{
*Correspondence to: Raymond Romano, Vanderbilt University School of Nursing, 46121 st Ave. S, Nashville, TN 37240, USA. Tel.: +1 615875 7690; Fax: +1 615343 7788; E-mail: Raymond. romano@vanderbilt.edu.
}

among those, an estimated 50-93\% will experience chronic pain [3-6]. Dementia is a known risk factor for the under-treatment of pain [6-9]. People with Alzheimer's disease (AD) receive less pain medication for similarly painful conditions when compared with cognitively intact older adults $[10,11]$. Pain is a multidimensional experience arising from cognitive, sensory, and affective central nervous system pro- 
cesses involving multiple neuronal circuits and brain regions.

Furthermore, AD impacts brain structure, such as the hypothalamus and the prefrontal cortex which is responsible for affective pain [6, 12-14]. A potential cause of the under-treatment of pain in dementia patients is the lack of understanding about how dementia influences the psychophysiological factors of the pain experience [6] and how these influences differ by sex in communicative people across the cognitive spectrum. Pain is the primary reason people seek medical attention, and more evidence is needed to understand the altered pain experience in $\mathrm{AD}$ patients as the prevalence of $\mathrm{AD}$ and related dementias is expected to triple over the next 30 years $[1,2,15]$.

Psychophysical studies of pain frequently report sex-related differences in the pain experience in cognitively healthy adults [16-18]. These sex differences may change with age since the prevalence of chronic pain increases with age as the function of endogenous pain inhibitory systems declines [19-21]. In cognitively healthy older adults, women tend to experience pain at a higher intensity and report pain more frequently than men [15, 22]. However, this relationship has not been demonstrated to exist across the cognitive spectrum.

Understanding patterns of pain responsivity across age, sex, and cognitive ability are essential given evidence of sex-associated differences in the psychophysical and neuropsychological processing of pain, the increased incidence of chronic pain in older adults, and the potential for undertreatment of pain in dementia. We have published several papers from the parent data set showing sex differences in cognitively healthy older adults [23]. We have also shown sex differences in a pilot sample of people with dementia only [24]. To extend upon this work, we now examine sex differences in pain across the cognitive spectrum. While several between-groups psychophysical studies on sex differences in pain appear in literature, there is a paucity of experimental pain research using a continuous measure of cognitive ability to predict pain outcomes across the cognitive spectrum. Understanding patterns of experimental pain responsivity across age, sex, and cognitive ability are essential given evidence of sex-associated differences in the psychophysical and neuropsychological processing of pain. This understanding may help to develop more effective treatment plans for the growing number of older adults experiencing chronic pain with comorbid dementia. Thus, we tested the hypothesis that sex differences in psychophysical processing of pain are significantly associated with increasing or decreasing levels of global cognition.

\section{METHODS}

\section{Study design}

This study is a secondary analysis of a crosssectional parent study designed to compare the psychological response to thermal pain in older adults with and without dementia. Thus, the current methods have been previously published [23-26]. The purpose of the current study was to examine sex differences in the association of cognitive impairment and thermal pain perception in verbally communicative older adults. Cognitive impairment, as assessed by the Mini-Mental State Examination (MMSE; range 0-30), was used as the primary covariate in our outcome analysis and included scores across the cognitive spectrum (no cognitive impairment to severe cognitive impairment).

The Vanderbilt University Institutional Review Board approved the parent study, and each participant, or legal surrogate when appropriate, provided written informed consent at the time of enrollment. Capacity to consent was assessed using the University of California San Diego Brief Assessment of Capacity to Consent (UBACC), that takes less than 5 minutes to complete. A UBACC score higher than 14.5 was required for informed consent, as this cut-off is $89 \%$ sensitive and $100 \%$ specific for determining capacity to consent for research [27]. Individuals with UBACC scores less than or equal to 14.5 (i.e., those lacking decisional capacity) signed an assent document and a legal surrogate provided consent. All participants and legal surrogates who completed the study were compensated US $\$ 100$ for their time.

\section{Participants}

The recruitment and enrollment protocol of the parent study are previously published [23-26]. Participants 65 years of age or older were recruited from the greater Nashville, Tennessee metropolitan area and enrolled over three years from 2012 to 2015. A clinical diagnosis of AD was verified based on documentation in the medical record of 1) neuropsychiatric evaluation, diagnostic MRI or PET, lack of other potential causes of memory loss; and 2) results of the evaluation with the MMSE, Montreal Cognitive Assessment, or the Functional Assessment 
Staging Scale. Cognitively healthy participants were recruited using a mass email, flyers, and recruitment presentations in healthcare facilities and local events.

Participants were excluded for the following reasons: the presence of a chronic pain diagnosis, daily use of opioid or non-opioid pain medication, upper extremity peripheral neuropathy, any current cancer diagnosis or treatment, previous stroke with residual deficit, Raynaud's Disease, unstable cardiac conditions, insulin-dependent diabetes, or current diagnosis of major depression.

Because the parent study included neuroimaging, additional exclusion criteria included claustrophobia, the presence of a pacemaker, ventricular shunt, or any implanted metal object not confirmed as 3 Tesla MRI compatible, multiple metal implants in the same extremity, or presence of movement disorders (e.g., Parkinson's disease, restless leg syndrome). Participants were required to be verbally communicative and able to provide a verbal pain rating.

\section{Screening and enrollment}

A two-part screening process included an initial telephone screening, followed by a one-hour visit to confirm eligibility either at the participant's place of residence or at the Vanderbilt University Medical Center. Before any data collection occurred, participants and the legal surrogate (when necessary) were allowed to experience the thermal pain stimulus and complete two practice psychophysics trials. Practice trials ensured that participants understood the directions and could respond appropriately. Evidence of an appropriate response included the ability to follow directions and vocalize pain.

\section{Measures}

A trained research assistant administered all study measures orally to participants to decrease subject and caregiver burden and to minimize missing data. Demographic measures included a detailed list of all medications and the Hollingshead Four-Factor Index of Socioeconomic Status (SES) [28]. The Brief Pain Inventory Short Form (BPI-SF) was used to collect current and average daily pain [29]. Depression and anxiety screens included the Geriatric Depression Scale-Short Form (GDS-15) [30] and the state and trait forms of the Spielberger State-Trait Anxiety Inventory (STAI) [31], respectively.

\section{Thermal stimulation protocol}

Trained research assistants administered the psychophysical thermal stimulation assessment over approximately 30 minutes following a modified protocol of the experimental mechanical pressure pain protocol by Cole et al. [13]. Two aspects of pain were assessed using the Medoc Pathway Pain and Sensory Evaluation System: the intensity, how strong the pain feels; and the unpleasantness, how unpleasant or disturbing the pain feels. A $30 \times 30 \mathrm{~mm}$ Medoc thermode was attached to the thenar eminence of the right hand. The thermode was programmed to deliver a baseline temperature of $30^{\circ} \mathrm{C}$ with increasing heat at a rate of $1^{\circ} \mathrm{C} / \mathrm{s}\left(\max\right.$ temperature $\left.=48^{\circ} \mathrm{C}\right)$.

Participants were instructed to signal to stop the increasing heat when the participant felt 'warmth', 'mild pain', or 'moderate pain'. The temperatures at which each participant stopped the increasing heat at each percept were documented. Following the approach we have previously published [23-26], pain intensity was evaluated using a 0-20 sensory numeric rating scale with anchors "warmth $=0$ ", "mild pain $=5 "$, and "moderate pain $=11 "$ ". Unpleasantness was evaluated using a 0-20 unpleasantness scale with anchors " $0=$ neutral", " $5=$ slightly unpleasant", " $8=$ unpleasant", " $11=$ very unpleasant", " $16=$ intolerable", and " $20=$ extremely distressing." Participants were acclimated to the scales and then completed three trials at each temperature condition, and the average self-reported pain intensity and unpleasantness ratings across the trials used in our analyses.

\section{Statistical analysis}

Participant characteristics and study data collected on a continuous scale were summarized using median and inter-quartile range (IQR) due to lack of normality (Fischer test $> \pm 2.58$ ). Frequency distributions summarized nominal and ordinal data. Comparisons between males and females were conducted using Mann-Whitney U tests (continuous) and Chi-Square Tests of Independence (nominal, ordinal). Cohen's d effect size indices were generated to summarize the magnitude of the gender effects. Associations of global cognition (MMSE scores) with the psychophysical reports (temperatures and ratings of unpleasantness) for males and females were generated using Pearson correlations. Differences between males and females in the strength and/or direction of those associations were tested 
using linear regressions. Each of the gender and MMSE distributions were mean centered and multiplied to create an interaction term that was entered into each regression, along with gender and MMSE as main effects, to test for differences between males and females in the association of MMSE with the respective psychophysical report used in the regression.

Before conducting these correlations and regressions, the skewed psychophysical distributions were transformed to normal. Such that at the warmth threshold, both temperature and unpleasantness values were rank transformed and at the mild and moderate pain thresholds, unpleasantness values were square-root transformed. For all analyses, a maximum alpha of $0.05(p<0.05)$ was used for determining statistical significance.

\section{RESULTS}

\section{Demographics}

A total of 80 participants were in the parent study. For this analysis, three participants were excluded due to an MMSE score of less than 10 and one subject was excluded due to missing an MMSE score. The final sample consisted of males $(n=38)$ and females $(n=38)$ with a median age of 73 (IQR: 68-80). MMSE scores ranged from 11 to 30 (IQR: 20-30). Of the sample $84 \%$ were white. On the day of the study, very few participants reported any pain (median both average and now $=0$ ) and fewer than $10 \%$ reported pain levels of greater than 2 ( 7 of $76,9 \%)$. There were no statistically significant differences between males and females on any of the participant characteristics investigated including most importantly the distribution of MMSE scores (see Table 1).

\section{Psychophysical}

Summaries of the psychophysical self-report data for males and females are in Table 3. Females reported mild and moderate pain at statistically significantly lower temperature values than did males $(p<0.05$, Cohen's $d=0.51-0.54)$. Males, on the other hand, rated the experience of moderate pain statistically significantly more unpleasant than did the females ( $p=0.016$, Cohen's $d=0.57$ ).
Table 1

Demographic and clinical characteristics of the participants

\begin{tabular}{|c|c|c|c|c|}
\hline & $\begin{array}{c}\text { Total } \\
(\mathrm{N}=76)\end{array}$ & $\begin{array}{l}\text { Female } \\
(n=38)\end{array}$ & $\begin{array}{c}\text { Male } \\
(n=38)\end{array}$ & $p$ \\
\hline Race & $\mathrm{N}(\%)$ & $\mathrm{N}(\%)$ & $\mathrm{N}(\%)$ & $\overline{0.529}$ \\
\hline \multirow[t]{2}{*}{ White } & $64(84)$ & $31(82)$ & $33(878)$ & \\
\hline & \multicolumn{4}{|c|}{ Median [IQR]Median [IQR]Median [IQR] } \\
\hline Age & $73[68,80]$ & $73[68,80]$ & $73[67,81]$ & 0.731 \\
\hline $\mathrm{MMSE}^{1}$ & $28[20,30]$ & $27[21,30]$ & $28[18,30]$ & 0.887 \\
\hline BPI Average $^{2}$ & $0[0,2]$ & $0[0,3]$ & $0[0,2]$ & 0.298 \\
\hline BPI Now ${ }^{2}$ & $0[0,0]$ & $0[0,0]$ & $0[0,0]$ & 0.893 \\
\hline $\mathrm{GDS}^{3}$ & $1[0,4]$ & $2[0,4]$ & $1[0,3]$ & 0.929 \\
\hline STAI State ${ }^{4}$ & $48[44,50]$ & $50[45,53]$ & $47[45,50]$ & 0.105 \\
\hline STAI Trait ${ }^{4}$ & $47[44,50]$ & $47[43,50]$ & $47[44,49]$ & 0.877 \\
\hline $\mathrm{SES}^{5}$ & $54[41,61]$ & $53[38,58]$ & $55[45,62]$ & 0.324 \\
\hline
\end{tabular}

${ }^{1}$ MMSE, Folstein Mini Mental State Examination (range $=0-30$; $0=$ completely cognitively impaired $30=$ completely cognitively intact). ${ }^{2}$ BPI-SF, Brief Pain Inventory Short Form (range = 0-10; $0=$ no pain, $10=$ most pain). ${ }^{3}$ GDS-SF, Geriatric Depression Scale Short Form (range; $0=$ no indication of depression, $15=$ high possibility of depression); Female: $n=37$, Male: $n=37$. ${ }^{4}$ STAI, Spielberger State or Trait Anxiety Inventory (range; $20=$ indicates increased anxiety, $80=$ indicates least amount of anxiety); Female: $n=34$, Male: $n=32 .{ }^{5}$ Hollingshead Four Factor Measure of SocioEconomic Status (range $=8-66 ; 8=$ lowest SES, $66=$ highest SES) Female: $n=38$, Male: $n=36$.

Table 2

Distribution of MMSE by gender groups

\begin{tabular}{lcc}
\hline MMSE & Female & Male \\
\hline & $(n=38)$ & $(n=38)$ \\
Cognitively Intact (29-30) & 14 & 17 \\
MCI (25-28) & 8 & 6 \\
Mild (20-24) & 9 & 5 \\
Moderate (15-19) & 1 & 6 \\
Moderately Severe (11-15) & 6 & 4 \\
\hline
\end{tabular}

MMSE, Mini-Mental State Examination (range $=0-30$; $0=$ completely cognitively impaired $30=$ completely cognitively intact). MCI, mild cognitive impairment.

Table 3

Psychophysics of temperature thresholds necessary to produce warmth, mild pain, or moderate pain and unpleasantness ratings at each condition

\begin{tabular}{lcccc}
\hline & Female & Male & \multicolumn{2}{c}{ Cohen's } \\
& $\begin{array}{c}(n=38) \\
\text { Median [IQR] }\end{array}$ & $\begin{array}{c}(n=38) \\
\text { Median [IQR }]\end{array}$ & $p$ & $d$ \\
\hline Temperature & & & & \\
$\quad$ Warmth & $33[32,35]$ & $33[32,36]$ & 0.139 & 0.34 \\
Mild pain & $36[35,40]$ & $39[35,44]$ & 0.022 & 0.54 \\
$\quad \begin{array}{l}\text { Moderate pain } \\
\text { Unpleasantness }\end{array}$ & $39[38,43]$ & $44[37,46]$ & 0.029 & 0.51 \\
$\quad$ Warmth & $0[0,1]$ & $0[0,2]$ & 0.115 & 0.30 \\
Mild pain & $3[0,5]$ & $4[1,6]$ & 0.140 & 0.33 \\
Moderate pain & $5[2,8]$ & $7[5,11]$ & 0.016 & 0.57 \\
\hline
\end{tabular}

Temperature was measured in degrees Celsius (range $=30-55^{\circ} \mathrm{C}$ ). Unpleasantness was measured by numerical descriptor scale $(0=$ neutral, $20=$ extremely unpleasant $)$. 


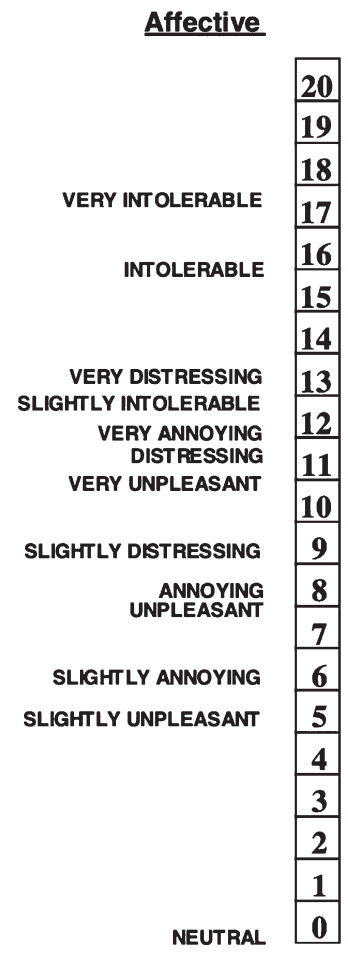

Sensory

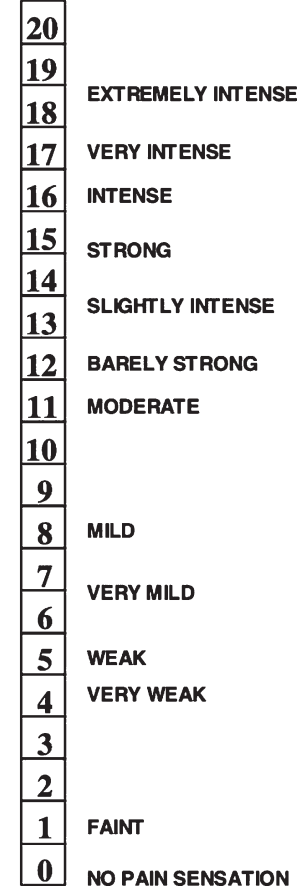

Fig. 1. Affective Sensory and Unpleasantness Numerical Scale. Numerical descriptor scale used to measure affective unpleasantness and sensory intensity. Reprinted with permission from Eur J Pain, 9, Petzke F, Harris RE, Williams DA, Clauw DJ, Gracely $\mathrm{RH}$, Differences in unpleasantness induced by experimental pressure pain between patients with fibromyalgia and healthy controls. 325-335. Copyright (2005), with permission from John Wiley and Sons.

\section{Associations of MMSE with psychophysical reports}

Associations of the participant MMSE scores with their psychophysical reports (temperature and unpleasantness) are summarized for the entire sample and by sex in Table 4 . A statistically significant inverse association of MMSE with temperature reports at the warmth threshold was observed for both males and females ( $r=-0.33$ and -0.41 respectively, $p<0.05)$ with no statistically significant difference between them $(p=0.767)$. Thus, regardless of sex, temperatures at which a warmth threshold was reported increased as MMSE scores decreased. Minimal associations were detected between MMSE scores and temperature reports at either of the other two thresholds for both sex groups ( $r=-0.13$ to -0.05 , $p>0.50$, see Table 4).

There was a statistically significant difference between males and females in the direction of the association of MMSE scores with unpleasantness ratings at the moderate pain threshold $(p=0.033)$. In females, the direction of the association was that ratings of unpleasantness increased as MMSE scores increased $(r=0.30, p=0.072)$. To the contrary, in males the direction of the association was reversed; ratings of unpleasantness at that threshold increased as MMSE scores decreased $(r=-0.20$, $p=0.221$ ). Thus, while neither of the specific correlations within each sex group was statistically

Table 4

Correlations of MMSE scores with temperature and unpleasantness ratings at each threshold level for the entire sample and within gender groups

\begin{tabular}{lcccc}
\hline & $\begin{array}{c}\text { Overall } \\
(\mathrm{N}=76)\end{array}$ & $\begin{array}{c}\text { Female } \\
(n=38)\end{array}$ & $\begin{array}{c}\text { Male } \\
(n=38)\end{array}$ & $\begin{array}{c}\text { Difference } \\
p^{*}\end{array}$ \\
\hline Temperature & $r(p)$ & $r(p)$ & $R(p)$ & \\
$\quad$ Warmth & $\mathbf{- 0 . 3 6 ( \mathbf { 0 . 0 0 1 } )}$ & $\mathbf{- 0 . 4 1 ( \mathbf { 0 . 0 1 0 } )}$ & $\mathbf{- 0 . 3 3 ( \mathbf { 0 . 0 4 4 } )}$ & 0.767 \\
$\quad$ Mild pain & $-0.09(0.460)$ & $-0.13(0.442)$ & $-0.07(0.671)$ & 0.959 \\
$\quad \begin{array}{l}\text { Moderate pain } \\
\text { Unpleasantness }\end{array}$ & $-0.08(0.482)$ & $-0.05(0.766)$ & $-0.11(0.508)$ & 0.720 \\
$\quad$ & & & \\
$\quad$ Warmth & $-0.01(0.990)$ & $-0.04(0.833)$ & $0.01(0.953)$ & 0.857 \\
$\quad$ Mild pain & $-0.07(0.543)$ & $0.07(0.698)$ & $-0.19(0.249)$ & 0.265 \\
Moderate pain & $0.03(0.772)$ & $\mathbf{0 . 3 0}(\mathbf{0 . 0 7 2})$ & $\mathbf{- 0 . 2 0}(\mathbf{0 . 2 2 1})$ & $\mathbf{0 . 0 3 3}$ \\
\hline
\end{tabular}

Associations of the participant MMSE scores with their psychophysical reports (temperature and unpleasantness). Skewed distributions were transformed to normal for Pearson correlations ( $r$ ) and linear regressions. Warmth threshold: Both temperature and unpleasantness values were rank transformed. Mild and moderate pain thresholds: Unpleasantness values were square-root transformed. ${ }^{*}$ Centered sex*MMSE interaction effect after controlling for main effects of sex and MMSE in linear regression analysis. 
significant, the magnitude of the difference between them in terms of the direction (positive or inverse) was statistically significant (interaction term, $p=0.033$ ). None of the other associations between MMSE scores and unpleasantness reports at either of the other two thresholds (warmth or mild pain) for both sex groups were statistically significant $(r=-0.19$ to $+0.07, p>0.20$ ) nor were any of the tests of difference between those correlations (see Table 4).

\section{DISCUSSION}

We found in both males and females that those with a lower global cognition rating required a higher temperature to elicit the perception of pain and males tended to report greater unpleasantness. This result parallels our previously reported pilot study [24] from this sample in which males with AD only, reported pain as more unpleasant. This study extends these findings in individuals across the cognitive spectrum. In parent study, males reported pain as more unpleasant regardless of cognitive status. These results are important because they demonstrate that sex differences in the perception of pain might be present across the cognitive spectrum.

Our finding that a greater pain stimulus is required to elicit the perception of pain with worsening cognition agrees with several previous studies from our laboratory and others $[7,8,12,13,32]$. As mentioned above, we have previously shown using the same methods described in this paper that people with a clinical diagnosis of AD demonstrated higher thermal thresholds for the detection of "warmth", "mild pain", and "moderate pain" [23]. Others have found that people with AD demonstrated higher mechanical pressure pain thresholds and increased detection thresholds for electrical shock pain threshold [13, 33], respectively. Regarding sex effects on the detection of thermal pain stimuli unlike previous studies, the current study showed no difference between males and females in pain stimuli required to elicit the perception of pain. Interestingly, while controlling for covariates, the detection of thermal pain may be driven by possible depression (based on MMSE screening) in males.

Our results of the affective response to pain were unlike those reported for younger adults; males show greater pain-associated unpleasantness than females $[15,22]$. The current study found evidence to suggest that in males worsening global cognitive function was associated with higher reports of unpleasantness in the presence of weak and moderate pain. This finding supports Benedetti and colleagues [34], who reported increased tolerance to highly unpleasant evoked pain stimuli (electrical shock and ischemic arm pain) in people with worsening AD.

As an exploratory secondary analysis, there are some obvious limitations in the current study to consider when interpreting results. The parent study was not powered to detect sex differences, and the sample size was moderate. Other sensory modalities (e.g., somatosensory, auditory) may contribute to the overall pain experience which was not in the scope of this paper. As we have previously noted [26], a perceptual matching paradigm was used rather than a fixed temperature for thermal sensory detection levels. This procedure may have impacted the pattern of findings for pain unpleasantness in unknown ways. To comply with IRB recommendation, we used the Method of Limits program on the Medoc Pathways Model so that the individual (regardless of cognition) was in control of the perceptual pain stopping point.

For this reason, we were unable to collect any perception above the rating of "moderate pain", specifically avoiding the perception of "severe pain" or the perception of "pain tolerance". This design was developed based on the mechanical pressure paradigm used by Cole and colleagues [13] in their perceptual matching paradigm of people with AD. Despite these limitations, the current study adds to the limited number of clinical and experimental pain studies examining sex differences in altered pain response in people with cognitive impairment. Another strength of the design is that the authorship team includes authors with experience in the legal and ethical issues in pain research in people with cognitive impairment [35].

The clinical relevance of these findings suggests that, in either sex, the diminished ability to detect pain could lead to an increase in adverse outcomes, such as an increase in the length of hospitalization [36]. Additionally, the increased reporting of unpleasantness in males may increase the risk of suffering from pain in those with worsening cognition. Relative to cognitively healthy controls, people with worsening cognition generally have an altered response to clinical and experimental pain. Sex differences in pain reports compound the risk of increased pain in those with worsening cognition, especially when onedimensional pain intensity scales are used that do not measure pain affect.

In summary, the current study found that sex differences in pain experiences may be present in older 
adults with varying degrees of cognition. Future studies are needed to replicate these results and should include an examination of the response to fixed temperatures and neurophysiological basis of sex differences in pain processing in a wide range of ages and across the spectrum of cognitive functioning. In consideration of mounting evidence, we continue to advocate that clinicians consider using a multidimensional sensory and unpleasantness pain tool in clinical practice as males and females may ultimately report pain intensity and pain unpleasantness differently, regardless of cognition. Using pain intensity measures only may predispose to suffering by not capturing an essential dimension of pain.

\section{Acknowledgments}

This work was supported by The John A. Hartford Foundation, Mayday Fund, National Center for Advancing Translational Sciences of the National Institute of Health under Award Number UL1 TR000445, Vanderbilt Clinical and Translational Research Scholars Program, the NIH National Institute on Aging (1R21AG045735-01A1; 1K23AG-), Dr. Failla was supported in part from an NIMH training grant (T32-MH18921) during completion of this work the Office of Academic Affiliations, Department of Veterans Affairs, VA National Quality Scholars Program and with resources and the use of facilities at VA Tennessee Valley Healthcare System, Nashville TN, and the Jonas Center Jonas Scholar 2016-2018. The content is solely the responsibility of the authors and does not necessarily represent the official views of the National Institutes of Health.

Authors' disclosures available online (https:// www.j-alz.com/manuscript-disclosures/19-0142r2).

\section{REFERENCES}

[1] Hebert LE, Weuve J, Scherr PA, Evans DA (2013) Alzheimer disease in the United States (2010-2050) estimated using the 2010 census. Neurology 80, 1778-1783.

[2] Patterson C (2018) World Alzheimer Report 2018. The State of the Art of Dementia Research: New Frontiers. Alzheimer's Disease International, London, UK.

[3] Corbett A, Husebo BS, Achterberg WP, Aarsland D, Erdal A, Flo E (2014) The importance of pain management in older people with dementia. Br Med Bull 111, 139-148.

[4] Abdulla A, Bone M, Adams N, Elliott AM, Jones D, Knaggs R, Martin D, Sampson EL, Schofield P (2013) Evidencebased clinical practice guidelines on management of pain in older people. Age Ageing 42, 151-153.

[5] van Kooten J, Delwel S, Binnekade TT, Smalbrugge M, van der Wouden JC, Perez RS, Rhebergen D, Zuurmond WW,
Stek ML, Lobbezoo F, Hertogh CM, Scherder EJ (2015) Pain in dementia: Prevalence and associated factors: Protocol of a multidisciplinary study. BMC Geriatr 15, 29.

[6] Stubbs B, Thompson T, Solmi M, Vancampfort D, Sergi G, Luchini C, Veronese N (2016) Is pain sensitivity altered in people with Alzheimer's disease? A systematic review and meta-analysis of experimental pain research. Exp Gerontol 82, 30-38.

[7] Monroe TB, Carter MA, Feldt KS, Dietrich MS, Cowan RL (2013) Pain and hospice care in nursing home residents with dementia and terminal cancer. Geriatr Gerontol Int 13, 1018-1025.

[8] Monroe TB, Gore JC, Chen LM, Mion LC, Cowan RL (2012) Pain in people with Alzheimer disease: Potential applications for psychophysical and neurophysiological research. J Geriatr Psychiatry Neurol 25, 240-255.

[9] Paulson CM, Monroe T, Mion LC (2014) Pain assessment in hospitalized older adults with dementia and delirium. $J$ Gerontol Nurs 40, 10-15.

[10] Haasum Y, Fastbom J, Fratiglioni L, Johnell K (2012) Undertreatment of osteoporosis in persons with dementia? A population-based study. Osteoporos Int 23, 1061-1068.

[11] Haasum Y, Fastbom J, Fratiglioni L, Kareholt I, Johnell K (2011) Pain treatment in elderly persons with and without dementia: A population-based study of institutionalized and home-dwelling elderly. Drugs Aging 28, 283-293.

[12] Scherder EJ, Sergeant JA, Swaab DF (2003) Pain processing in dementia and its relation to neuropathology. Lancet Neurol 2, 677-686.

[13] Cole LJ, Farrell MJ, Duff EP, Barber JB, Egan GF, Gibson SJ (2006) Pain sensitivity and fMRI pain-related brain activity in Alzheimer's disease. Brain 129, 2957-2965.

[14] Jones AK, Kulkarni B, Derbyshire SW (2003) Pain mechanisms and their disorders. Br Med Bull 65, 83-93.

[15] Johannes CB, Le TK, Zhou X, Johnston JA, Dworkin RH (2010) The prevalence of chronic pain in United States adults: Results of an Internet-based survey. J Pain 11, 12301239.

[16] Straube T, Schmidt S, Weiss T, Mentzel HJ, Miltner WH (2009) Sex differences in brain activation to anticipated and experienced pain in the medial prefrontal cortex. Hum Brain Mapp 30, 689-698.

[17] Paulson PE, Minoshima S, Morrow TJ, Casey KL (1998) Gender differences in pain perception and patterns of cerebral activation during noxious heat stimulation in humans. Pain 76, 223-229.

[18] Fillingim RB, King CD, Ribeiro-Dasilva MC, RahimWilliams B, Riley JL 3rd (2009) Sex, gender, and pain: A review of recent clinical and experimental findings. J Pain 10, 447-485.

[19] Edwards RR, Fillingim RB, Ness TJ (2003) Age-related differences in endogenous pain modulation: A comparison of diffuse noxious inhibitory controls in healthy older and younger adults. Pain 101, 155-165.

[20] Naugle KM, Cruz-Almeida Y, Fillingim RB, Riley JL 3rd (2013) Offset analgesia is reduced in older adults. Pain 154, 2381-2387.

[21] Riley JL 3rd, King CD, Wong F, Fillingim RB, Mauderli AP (2010) Lack of endogenous modulation and reduced decay of prolonged heat pain in older adults. Pain 150, 153-160.

[22] Leveille SG, Zhang Y, McMullen W, Kelly-Hayes M, Felson DT (2005) Sex differences in musculoskeletal pain in older adults. Pain 116, 332-338.

[23] Monroe TB, Gore JC, Bruehl SP, Benningfield MM, Dietrich MS, Chen LM, Newhouse P, Fillingim R, Chodkowski 
B, Atalla S, Arrieta J, Damon SM, Blackford JU, Cowan RL (2015) Sex differences in psychophysical and neurophysiological responses to pain in older adults: A cross-sectional study. Biol Sex Differ 6, 25.

[24] Cowan RL, Beach PA, Atalla SW, Dietrich MS, Bruehl SP, Deng J, Wang J, Newhouse PA, Gore JC, Monroe TB (2017) Sex differences in the psychophysical response to contact heat in moderate cognitive impairment Alzheimer's disease: A cross-sectional brief report. J Alzheimers Dis 60, 16331640.

[25] Monroe TB, Beach PA, Bruehl SP, Dietrich MS, Rogers BP, Gore JC, Atalla SW, Cowan RL (2017) The impact of Alzheimer's disease on the resting state functional connectivity of brain regions modulating pain: A cross sectional study. J Alzheimers Dis 57, 71-83.

[26] Monroe TB, Gibson SJ, Bruehl SP, Gore JC, Dietrich MS, Newhouse P, Atalla S, Cowan RL (2016) Contact heat sensitivity and reports of unpleasantness in communicative people with mild to moderate cognitive impairment in Alzheimer's disease: A cross-sectional study. BMC Med 14, 74.

[27] Jeste DV, Palmer BW, Appelbaum PS, Golshan S, Glorioso D, Dunn LB, Kim K, Meeks T, Kraemer HC (2007) A new brief instrument for assessing decisional capacity for clinical research. Arch Gen Psychiatry 64, 966-974.

[28] Hollingshead A (1975) Four factor index of social status. Yale University, New Haven.
[29] Cleeland CS, Ryan KM (1994) Pain assessment: Global use of the Brief Pain Inventory. Ann Acad Med Singapore 23, 129-138.

[30] Kurlowicz L (1999) The Geriatric Depression Scale (GDS). Geriatr Nurs 20, 212-213.

[31] Spielberger CD, Gorsuch RL, Lushene RE (1970) Manual for the State-Trait Anxiety Inventory. In Consulting Psychologists Press, Palo Alto, CA.

[32] Kunz M, Scharmann S, Hemmeter U, Schepelmann K, Lautenbacher $S$ (2007) The facial expression of pain in patients with dementia. Pain 133, 221-228.

[33] Gibson SJ, Voukelatos X, Ames D, Flicker L, Helme RD (2001) An examination of pain perception and cerebral event-related potentials following carbon dioxide laser stimulation in patients with Alzheimer's disease and agematched control volunteers. Pain Res Manag 6, 126-132.

[34] Benedetti F, Vighetti S, Ricco C, Lagna E, Bergamasco B, Pinessi L, Rainero I (1999) Pain threshold and tolerance in Alzheimer's disease. Pain 80, 377-382.

[35] Monroe TB, Herr KA, Mion LC, Cowan RL (2013) Ethical and legal issues in pain research in cognitively impaired older adults. Int J Nurs Stud 50, 1283-1287.

[36] Morrison RS, Magaziner J, McLaughlin MA, Orosz G, Silberzweig SB, Koval KJ, Siu AL (2003) The impact of post-operative pain on outcomes following hip fracture. Pain 103, 303-311. 